Epidemiology and Infection

http://journals.cambridge.org/HYG

EPIDEMIOLOGY \&

INFECTION

Additional services for Epidemiology and Infection:

Email alerts: Click here

Subscriptions: Click here

Commercial reprints: Click here

Terms of use : $\underline{\text { Click here }}$

Prevalence estimates of diagnosed viral hepatitis $B$, liver condition outcomes and hospitalization costs: a population record-linkage study in Tayside, Scotland

D. J. McLERNON, P. T. DONNAN and J. F. DILLON

Epidemiology and Infection / FirstView Article / January 2006, pp 1 - 9

DOI: 10.1017/S095026881200266X, Published online:

Link to this article: http://journals.cambridge.org/abstract_S095026881200266X

How to cite this article:

D. J. MCLERNON, P. T. DONNAN and J. F. DILLON Prevalence estimates of diagnosed viral hepatitis B, liver condition outcomes and hospitalization costs: a population record-linkage study in Tayside, Scotland. Epidemiology and Infection, Available on CJO doi:10.1017/S095026881200266X

Request Permissions : Click here 


\title{
Prevalence estimates of diagnosed viral hepatitis $B$, liver condition outcomes and hospitalization costs: a population record-linkage study in Tayside, Scotland
}

\author{
D. J. MCLERNON ${ }^{1 *}$, P. T. DONNAN ${ }^{2}$ AND J. F. DILLON $\operatorname{LON}^{3}$ \\ ${ }^{1}$ Division of Applied Health Sciences, University of Aberdeen, UK \\ ${ }^{2}$ Division of Population Health Sciences, University of Dundee, UK \\ ${ }^{3}$ Biomedical Research Institute, University of Dundee, UK
}

Received 7 December 2011; Final revision 11 October 2012; Accepted 2 November 2012

\section{SUMMARY}

We estimated prevalence and incidence of liver condition outcomes, and costs to the health service of diagnosed hepatitis B virus (HBV) in Tayside, UK. HBV patients were identified from electronic virology data between 1989 and 2003. The health resource costs of HBV for surface antigen-positive (HBsAg +) patients and $\mathrm{HBV}(\mathrm{HBsAg}+$ or immune, i.e. recovered) patients were calculated. A total of 633 patients had HBV $(275 \mathrm{HBsAg}+)$, and were more likely to be male ( $62 \%$ vs. $48 \%$ ), older (mean age $42 \cdot 6$ vs. $39 \cdot 2$ years) and deprived than the general population. The prevalence of immune individuals increased steadily. Post-HBV diagnosis, $24 \%$ of immune and $13 \%$ of HBsAg + patients were diagnosed with a liver condition. The median cost per immune patient $(£ 3023)$ was greater than per HBsAg + patient $(£ 1498)(P=0 \cdot 02)$. While increasing prevalence of immune HBV patients highlights an increase in screening and treatment, the costs associated with this group are high.

Key word: Hepatitis B.

\section{INTRODUCTION}

Hepatitis B virus (HBV) infection has a high prevalence worldwide. The World Health Organization estimated that about 2 billion people have been infected, and 350 million live with chronic infection [1]. The virus is responsible for 500000 to 1.2 million deaths per year [2]. Globally, $30 \%$ of cirrhosis and $78 \%$ of hepatocellular carcinoma (HCC) are attributable to HBV [3]. In Europe, it is estimated that 1 million people are exposed annually to $\mathrm{HBV}$, with

\footnotetext{
* Author for correspondence: Dr D. J. McLernon, Medical Statistics Team, Division of Applied Health Sciences, University of Aberdeen, Polwarth Building, Foresterhill, Aberdeen AB25 2ZD, UK.

(Email: d.mclernon@abdn.ac.uk)
}

22000 dying from cirrhosis and liver cancer due to the infection [4]. In the UK, a report by the Hepatitis B Foundation UK estimated that chronic hepatitis B infection almost doubled from 180000 in 2002 to 320000 in 2007 [5].

Due to the high morbidity and mortality associated with liver disease and HCC, the economic burden of HBV is considerable [2], with healthcare costs estimated at over three times higher for chronic hepatitis $\mathrm{B}$ than controls [6]. Ascertainment of the true incidence of liver diseases such as HBV is a difficult but crucial task that is required to adequately identify research needs and assess health resource implications.

This population-based retrospective cohort study aimed to estimate the crude prevalence and incidence rates of diagnosed HBV in Tayside, UK, describe the 
liver condition outcomes between patients with different stages of $\mathrm{HBV}$, and estimate the hospitalization costs associated with $\mathrm{HBV}$-infected patients.

\section{MATERIALS AND METHODS}

\section{Study population}

This study was carried out on people who were residents of Tayside, Scotland in the study period 1989-2003. Tayside is a mixed urban/rural region in the eastern side of the central belt of Scotland. It is representative of Scotland with regards to age, gender, ethnicity and unemployment, and has a population of approximately 429000 [7]. All patients who were ever diagnosed with HBV during the study period were identified from the virology dataset from Ninewells Hospital where all viral hepatitis tests in the Tayside region are performed, thus giving complete population coverage. Requests for viral hepatitis tests were from general practice (primary care), hospital (general admissions and specialist clinics) and institutional services (e.g. prison service).

Patient data were extracted and anonymized by the Health Informatics Centre (HIC) [8]. The databases relevant to this study, listed below, covered the entire study period and were used within procedures approved under the Data Protection Act and Caldicott Guardian with annual external audit.

(1) Virology database. Computerized patient-specific records exist for all requests for viral serology from primary and secondary care for hepatitis viruses for the Tayside region and all tests were extracted from the period 1989 to 2003.

(2) Scottish Morbidity Records (SMR01). This is a list of hospital admissions for all hospitals in Scotland. Type and dates of hospital admissions for viral hepatitis subjects were obtained from this data setfor Tayside.

(3) Epidemiology of Liver Disease In Tayside $(E L D I T)$. Contains all Tayside patients with liver diseases that have been 'electronically' diagnosed using record-linkage of biomedical datasets, including virology and immunology [9]. The database has been used for research projects such as the ALFIE study [10]. ELDIT was used to identify liver disease outcomes in HBV patients.

(4) Carstairs social deprivation categories. The Carstairs score is a socioeconomic measure based on data derived from the decennial census and linked to all residents in Tayside, via the postcode [11]. The score is divided into seven categories in order of deprivation where one is most affluent and seven is the most deprived.

All of the electronic databases described above were electronically linked using a unique identifier, the community health index (CHI) [12]. In Scotland, the $\mathrm{CHI}$ is assigned to every patient registered with a general practice and is contained within all of the databases described above. The CHI is a 10-digit number where the first six digits are the patient's data of birth, the seventh and eighth digits are a marker for gender, and the last two digits are check digits. Since the $\mathrm{CHI}$ contains person identifiable information, the data protection officer at HIC mapped it to a project specific anonymous ten-digit code and removed the CHI.

An algorithm was used to assign disease states. For most of the study period, HBV DNA was not available, so disease states are based on virological parameters extracted from the virology database. The algorithm, based on these limitations, assigned a patient's $\mathrm{HBV}$ as: acute if the surface antigen of the HBV (HBsAg) is positive $(+)$ and the hepatitis B core antibody immunoglobulin M (HBcAbIgM) + (at high titre, i.e. $\geqslant 50 \mathrm{IU} / 1$ ); chronic active infection if $\mathrm{HBsAg}+$, hepatitis B ' $\mathrm{e}$ ' antigen $(\mathrm{HBeAg})+$ and $\mathrm{HBcAbIgM}$ negative (low titre $<50 \mathrm{IU} / 1$ ); low infectivity carrier if $\mathrm{HBsAg}+$ and hepatitis B ' $\mathrm{e}$ ' antibody $(\mathrm{HBeAb})+$; and immune (i.e. recovered) if hepatitis $\mathrm{B}$ surface antibody (HBsAb) + and hepatitis B core antibody $(\mathrm{HBcAb})+$. This system means that some patients who are pre-core mutants will be included in the carrier group, but without the wider availability of HBV DNA in the earlier patients in the cohort this is unavoidable. The first date of each stage was used as date of diagnosis.

\section{Cost analysis}

The cost of hospitalization for viral hepatitis patients was calculated with 2009 net costs by speciality group for Ninewells Hospital, obtained from the Scottish Health Services Cost book on the Information Statistics Division website [13]. Although our study period ended in 2003, we used 2009 costs to reflect the current economic climate. The direct medical costs for hospitalized patients included hospital charges to house the patient in the hospital per day, laboratory testing, physician's fees, allied health professional 
staff and diagnostic procedures, but excluded both liver-specific and non-liver-specific medication costs. If a specific speciality group was not available at Ninewells Hospital, the average cost for all Scottish hospitals which did have the speciality was used. The median costs of hospitalization for immune patients and HBsAg + patients were compared using the Mann-Whitney test. The proportion of costs related to admission for liver disease was calculated. Liver disease admissions were identified from the International Classification of Diseases codes (versions 9 and 10) in the SMR01 database.

\section{Statistical analysis}

Crude estimates of yearly incidence and prevalence rates were based on the number of Tayside residents with newly diagnosed viral hepatitis B in each year from 1989 by stage and on those whose stage of hepatitis B changed during the study period. In calculating these estimated rates, the entire population of Tayside for the year was considered to be at risk. The denominator was estimated from the yearly General Register Office for Scotland population estimates. The rates from 1989 to 1998 have been reported in a previous study [14]. A dynamic population was used from 1989 to 2003 to account for migration. For the description of liver condition outcomes and cost analysis, a more fixed population was used where only patients who had always lived in Tayside from 1989 to 2003 or who moved into Tayside and remained there until the end of the study were included. This was because there was no way of knowing the exact date a patient left Tayside.

Kaplan-Meier curves were used to illustrate the time to liver condition diagnosis in hepatitis B patients for immune patients and carriers from the incident date of positive sample. Acute infected patients were not included because in reality these patients do not stay in this stage long and so there will be too few patients (and hence events) in these groups. However, they were included in the descriptive table of liver condition outcomes. Statistical analyses were performed using SAS version 9.3 (SAS Institute Inc., USA).

\section{RESULTS}

Among Tayside residents a total of 633 patients were identified with viral hepatitis B in the study period 1989-2003. Of these, 275 (43\%) patients were identified as HBsAg + or having once been $\mathrm{HBsAg}+$, and
$400(63 \%)$ were immune. Of the 633 patients, 42 seroconverted from $\mathrm{HBsAg}+$ to $\mathrm{HBsAb}+$ (i.e. immune) during observation, so 358 patients had previous infection but were immune either at point of testing for HBV or at first entry into the electronic database. Of the HBsAg + patients, 78 had acute infection. Of these, 47 had a different final recorded stage of HBV (30 immune, seven carrier, 10 chronic active). Of the HBsAg + patients, 48 had chronic active infection, of whom six became carriers and seven became immune. The descriptive analyses of the patients are given in Table 1. HBV patients were slightly older than the general population average and tended to live in less affluent areas. However, the mean age of chronic active patients was 5.6 years younger than the population average. HBV patients were more likely to be male compared to the general population.

The estimated incidence and prevalence of diagnosed viral hepatitis B infection by stage are displayed in Figure $1(a, b)$, respectively. Only estimated incidence is given for the diagnosed acute stage as this lasts for 6 months by definition. The estimated prevalence of diagnosed chronic active hepatitis B increased steadily each year until 1996, and then remained reasonably steady at around 4/100000 until 2003. The estimated prevalence of diagnosed hepatitis B carriers increased steadily until 1999 and remained reasonably constant between 26 and 28/100000 until 2003. The overall estimated prevalence of diagnosed HBsAg + rose to 37/100 000 in 1999 and stayed fairly steady. The estimated prevalence of diagnosed immune patients increased at a steady rate each year to $60 / 100000$ in 2003.

\section{Liver condition diagnosis}

Out of the 334 immune patients who were in the fixed Tayside population, 40 had a liver condition before their first recorded HBV test. Of these, 22 had HCV and 13 had alcohol-related liver disease. Post-HBV diagnosis, one quarter $(n=80)$ of the immune patients had a liver condition diagnosed (of whom only six had a diagnosis of a different liver condition before their first recorded HBV test) (see Table 2). From Figure 2 it is clear that the majority of liver conditions were diagnosed on the same day as the first HBV test. HCV was the most frequently diagnosed liver condition, infecting 64 (19\%) immune patients (including HCV in combination with other conditions). Only $10 \%$ of all diagnosed HBV carriers were later diagnosed with a liver condition. Four out of 22 patients were 


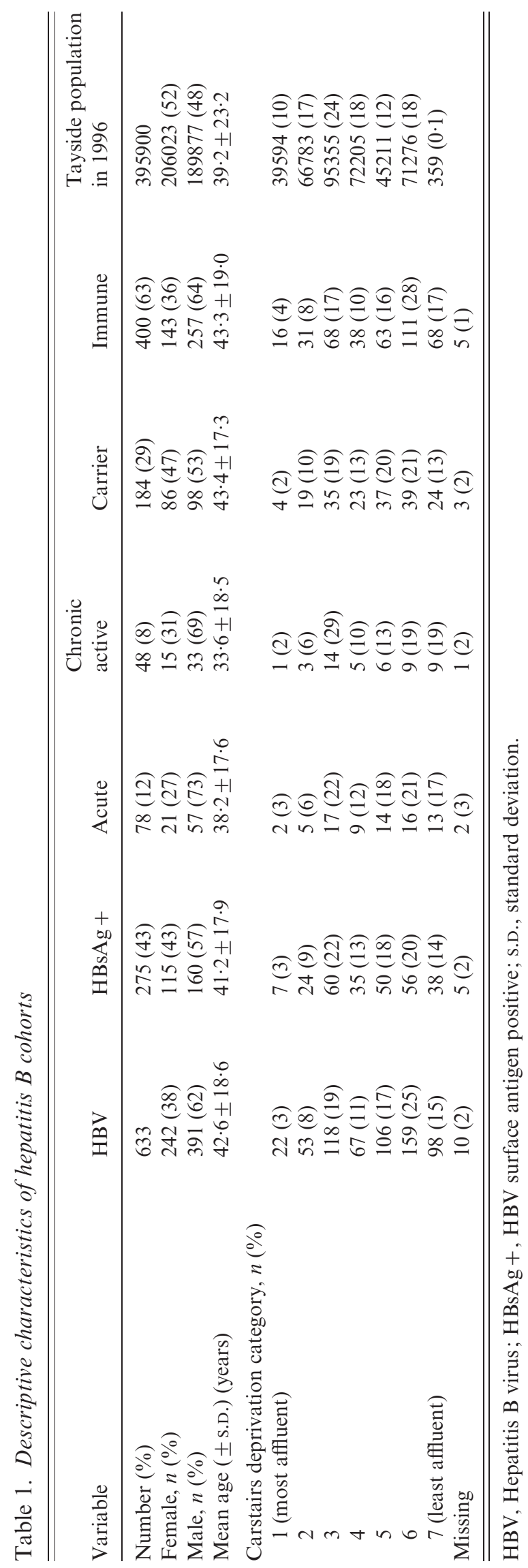

diagnosed with a liver condition while in the acute stage and 6/22 while in the chronic active stage.

\section{Cost analysis}

Table 3 shows the frequency of hospital admission, length of stay and cost analysis for HBV patients, and separately for HBsAg + and immune patients. The median cost per HBV patient admitted was $£ 8453$ and per HBV patient was $£ 2394$. The median cost per patient admitted $(P=0.02)$ and the median cost per patient $(P=0.02)$ were both significantly greater for immune patients than HBsAg + patients. However, there was no significant difference in the median length of stay between the two groups, which was 4 days. The proportion of the total hospitalization costs that were related to admissions for liver disease was $17 \cdot 1 \%$ ( $£ 1108078$ out of $£ 6480828$ ). For HBsAg + patients the proportion was $27 \cdot 6 \%$ ( $£ 374209$ out of $£ 1354865)$ and for immune patients the proportion was $14 \cdot 3 \%$ ( $£ 733870$ out of $£ 5125442$ ).

\section{DISCUSSION}

This study found that the prevalence of $\mathrm{HBsAg}+$ (i.e. acute, carrier, chronic) remained reasonably constant between 34 and 37 cases/100000 person-years from 1999 to 2003. The prevalence of immune patients rose steadily each year. Twelve percent of immune patients were diagnosed with a liver condition before their first recorded HBV test and a further $22 \%$ were diagnosed post-test. Of those diagnosed post-HBV test, $80 \%$ were diagnosed with $\mathrm{HCV}$. The median direct hospital costs per HBV immune patient were more than double the median costs per HBsAg + patient. Over a quarter of the costs for $\mathrm{HBsAg}+$ patients were related to admissions with liver disease compared to only $14 \%$ for immune patients.

However, these results should be interpreted in light of the underlying denominator of people tested for HBV in Tayside. The samples originated from all clinical services across Tayside, with the majority from hospital-based practice. While we do not have indications for testing in our database, we can speculate that the main reasons for testing include the presence of symptoms of acute hepatitis or chronic liver disease, being part of a broader work-up for other conditions and screening. The first two reasons most probably relate to those patients with acute or chronic active infection, while the latter is the most likely reason for testing in the immune group, due to 

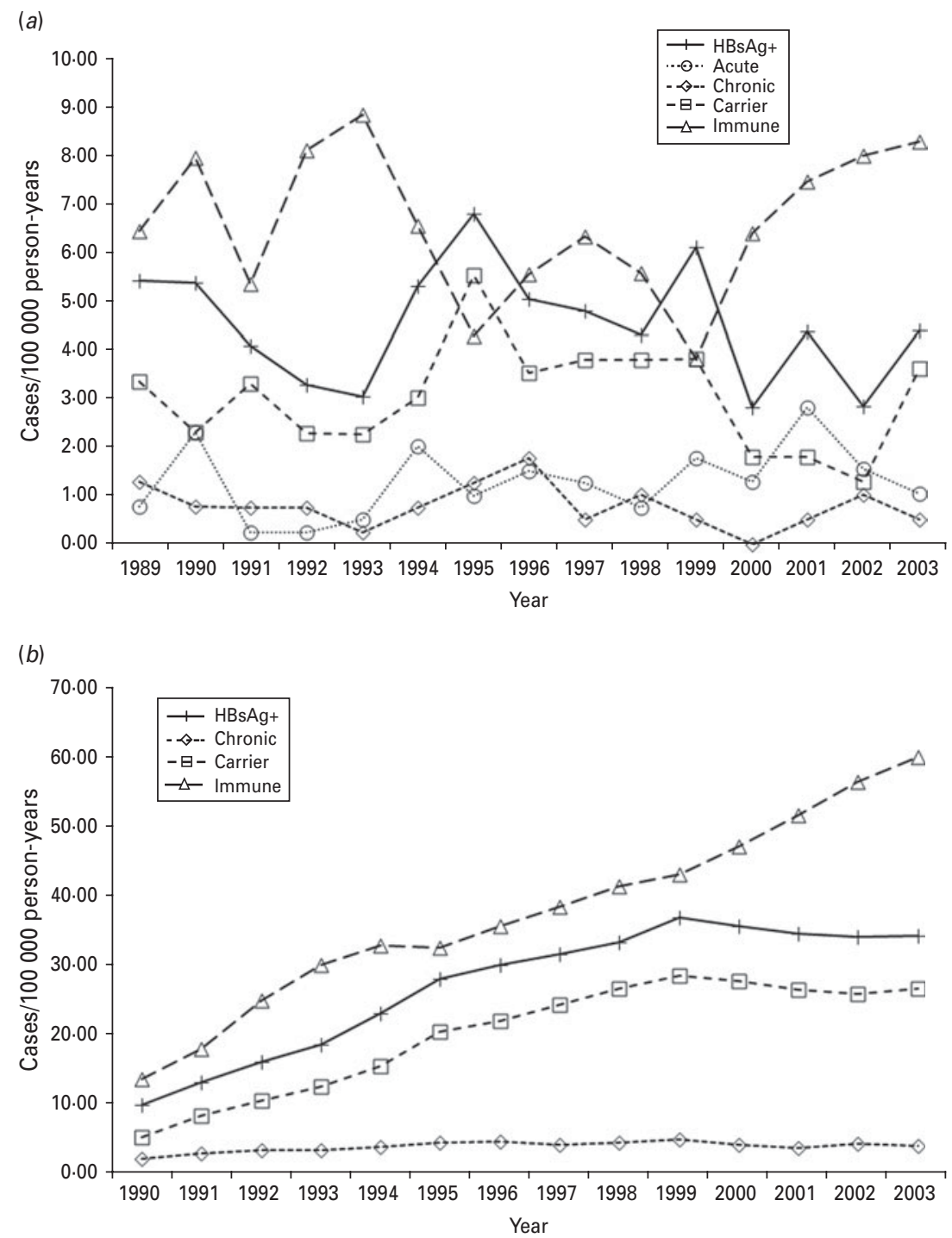

Fig. 1. (a) Estimated incidence and (b) prevalence of diagnosed hepatitis B in Tayside by stage of disease over 14 years.

these patients having a range of factors that are likely to increase the risk of a liver condition, e.g. injecting drug use. Those screened may also include pregnant women undergoing antenatal screening, which was introduced in Tayside in the early 1990s. Therefore it is important to note that the results found in patients tested for HBV may not be representative of the total population of patients who have HBV.

\section{Strengths and limitations}

Tayside is a mixed rural urban health board with varied socioeconomic status. The population in Tayside is predominantly caucasian with less than $2 \%$ immigrants [7]. Our electronic databases did not contain information on indications for testing or ethnic origin of HBV patients which could be major confounders for the disease. However, since immigrants from high-prevalence countries are a highrisk group for $\mathrm{HBV}$, we can assume that a higher proportion of immigrants make up the HBV population than the general population of Tayside. Since the percentage of immigrants in Tayside is similar to the whole of Scotland, our findings may be regarded as the background rate of diagnosed HBV infection and cost for Scotland. Clearly there will be other localities throughout the UK with higher rates of chronic HBV due to immigrants from endemic areas.

The study period ended in 2003 which may impact the relevance of the findings to the present with regard to changes in healthcare, treatment and immigration. Estimates of net migration in Tayside and throughout 
Table 2. Liver conditions diagnosed post-HBV diagnosis by HBV stage

\begin{tabular}{|c|c|c|c|c|c|}
\hline \multirow[b]{2}{*}{ Liver condition } & \multicolumn{5}{|c|}{ Stage of hepatitis B virus } \\
\hline & $\begin{array}{l}\text { Acute }(N=22) \\
n(\%)\end{array}$ & $\begin{array}{l}\text { Chronic active } \\
(N=22) n(\%)\end{array}$ & $\begin{array}{l}\text { Carrier } \\
(N=122) n(\%)\end{array}$ & $\begin{array}{l}\text { Immune }(N=334) \\
n(\%)\end{array}$ & $\begin{array}{l}\text { All }(N=500) \\
n(\%)\end{array}$ \\
\hline HCV only & $2(9)$ & $1(5)$ & $4(3)$ & $56(17)$ & $63(13)$ \\
\hline Cirrhosis only & $1(5)$ & $2(9)$ & $3(2)$ & $4(1 \cdot 2)$ & $10(2)$ \\
\hline HCC only & $1(5)$ & $1(5)$ & $1(1)$ & $1(0 \cdot 3)$ & $4(0 \cdot 8)$ \\
\hline ARLD only & 0 & $1(5)$ & 0 & $2(0 \cdot 6)$ & $3(0 \cdot 6)$ \\
\hline $\mathrm{HCV}+\mathrm{ARLD}$ & 0 & $1(5)$ & 0 & $2(0 \cdot 6)$ & $3(0 \cdot 6)$ \\
\hline $\mathrm{HCV}+$ cirrhosis $+\mathrm{ARLD}$ & 0 & 0 & $1(1)$ & $2(0 \cdot 6)$ & $3(0 \cdot 6)$ \\
\hline Alcoholic hepatitis only & 0 & 0 & 0 & $2(0 \cdot 6)$ & $2(0 \cdot 4)$ \\
\hline Hepatitis A virus only & 0 & 0 & 0 & $2(0 \cdot 6)$ & $2(0 \cdot 4)$ \\
\hline $\mathrm{HCV}+$ cirrhosis & 0 & 0 & $1(1)$ & $1(0 \cdot 3)$ & $2(0 \cdot 4)$ \\
\hline $\mathrm{HCV}+$ cirrhosis $+\mathrm{HCC}$ & 0 & 0 & 0 & $2(0 \cdot 6)$ & $2(0 \cdot 4)$ \\
\hline Cirrhosis + HCC & 0 & 0 & 0 & $2(0 \cdot 6)$ & $2(0 \cdot 4)$ \\
\hline ARLD + cirrhosis & 0 & 0 & 0 & $2(0 \cdot 6)$ & $2(0 \cdot 4)$ \\
\hline Autoimmune hepatitis only & 0 & 0 & 0 & $1(0 \cdot 3)$ & $1(0 \cdot 2)$ \\
\hline Fatty liver disease only & 0 & 0 & $1(1)$ & 0 & $1(0 \cdot 2)$ \\
\hline ARLD + HCC + cirrhosis & 0 & 0 & $1(1)$ & 0 & $1(0 \cdot 2)$ \\
\hline $\mathrm{HCV}+$ alcoholic hepatitis & 0 & 0 & 0 & $1(0 \cdot 3)$ & $1(0 \cdot 2)$ \\
\hline Any & $4(18)$ & $6(27)$ & $12(10)$ & $80(24)$ & $102(20)$ \\
\hline Time to diagnosis, median (IQR), days & $5(0-46 \cdot 5)$ & $37 \cdot 5(0-133)$ & $47(0-832)$ & $0(0-29 \cdot 5)$ & $0(0-71)$ \\
\hline
\end{tabular}

HCV, Hepatitis C virus; HCC, hepatocellular carcinoma; ARLD, alcohol-related liver disease; IQR, interquartile range. 


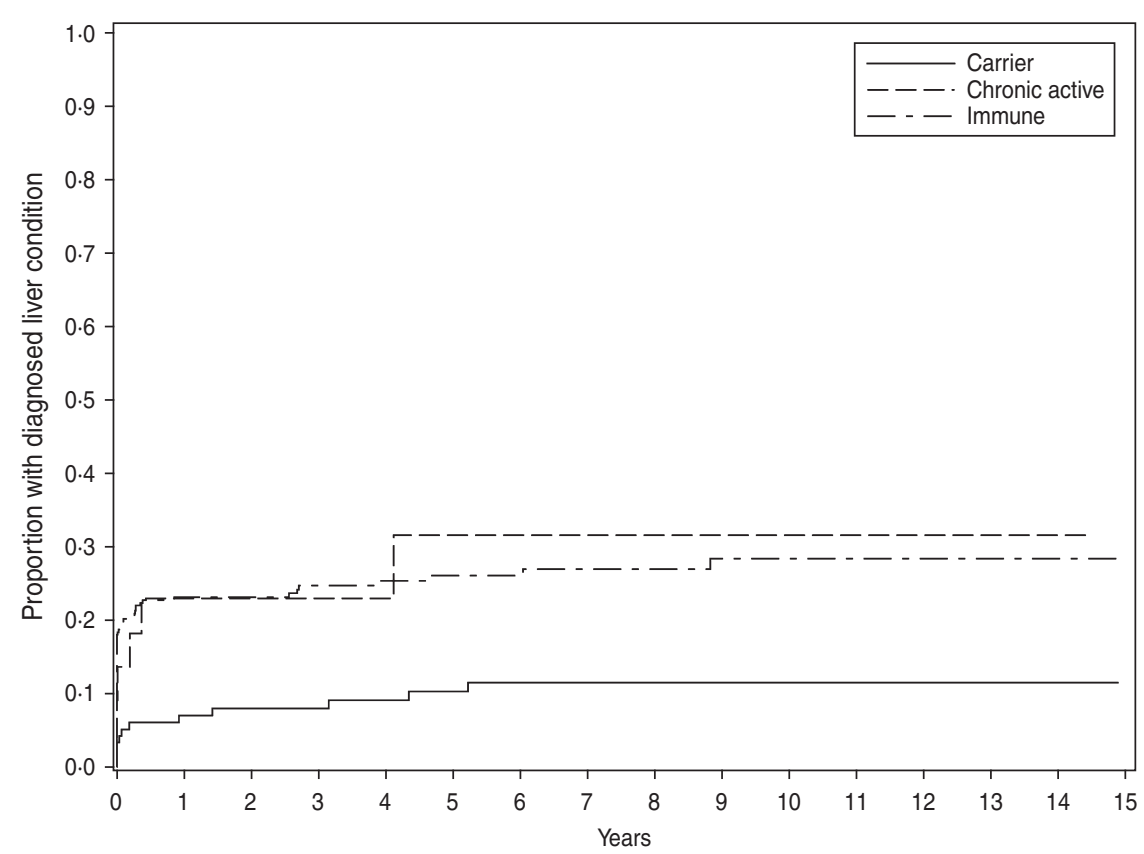

Fig. 2. Kaplan-Meier plot of time to liver disease diagnosis from first hepatitis B test for HBV chronic active infected patients, carriers and immune patients.

Scotland have increased yearly suggesting that the incidence and prevalence of carriers may have increased at a greater rate during the past 8 years [14]. With the advancement of HBV treatment and improvements in healthcare the lengths of stay in hospital may be shorter. However, with the increase in treatment and healthcare costs we would expect changes in the median cost per patient to be minimal. It is important to note that prevalence estimates of the diagnosis of HBV stage are strictly from 1989. We have no records of the 'base' prevalence before this period. The true incidence and prevalence of HBV diagnosis (regardless of stage) throughout the study period may well be slightly higher than estimated here since there may be a small percentage of error in Tayside residency data or laboratory data entry. Obviously, we would expect the true incidence and prevalence of $\mathrm{HBV}$ to be higher than our estimates since many individuals with acute infection are never tested, particularly asymptomatic patients.

This study extends previous work published on the prevalence and economic burden of hepatitis B on an earlier dataset [15], and, to our knowledge, remains the first population-based study that has recordlinked clinical data to identify viral hepatitis subjects from primary and secondary care. These results are derived from unselected 'real-world' observations in a geographically defined population. The database created for this study forms part of the ELDIT database [9]. ELDIT was created via the recordlinkage of various datasets including SMR01 hospital admissions, the SMR06 cancer registry, and hospital datasets containing virology, immunology, pathology, and endoscopy information. Each liver disease was electronically diagnosed using strict algorithms. Uniquely, in Tayside, record-linkage using the CHI number allows investigation of the whole area population to measure incidence and prevalence of diagnosed disease rather than depending on referral patterns with multiple in-built biases and faults.

A limitation of the study was the lack of an exact emigration date from Tayside for the whole study period meaning that censoring due to migration could not be accounted for in our survival and hospital admissions analyses. Only the year of emigration from Tayside was known. Therefore, only patients who resided in Tayside from their first recorded HBV test until the end of 2003 were included. Comparing the denominators in Tables 1 and 3 we observed that $66 /$ $400(16 \cdot 5 \%)$ immune patients and 109/275 (39.6\%) HBsAg + patients left Tayside before the end of the period. The larger proportion for the latter group is not surprising given that HBV carriers are predominantly immigrants who tend to change location more regularly than natives, who comprise the majority of the immune group. Another limitation is that the cost analysis only included direct medical costs and excluded both liver-specific and non-liver-specific 
Table 3. Hospital admission frequencies, length of stay and costs for HBsAg-positive patients, immune patients and all $H B V$ patients

\begin{tabular}{llll}
\hline \hline Hospital admission variable & HBsAg $+(N)$ & Immune $(N)$ & HBV $(N)$ \\
\hline (A) Number of patients & 166 & 334 & 500 \\
(B) Number of patients admitted & 102 & 222 & 324 \\
(C) Number of admissions & 300 & 1059 & 1359 \\
(D) No hospital admission reported & 64 & 112 & 176 \\
Average number of hospital admissions (C/A) & $1 \cdot 8$ & $3 \cdot 2$ & $2 \cdot 7$ \\
Re-admissions to hospital per patient (C-B)/B & $1 \cdot 9$ & $3 \cdot 8$ & $3 \cdot 2$ \\
Length of stay (LOS) & & & \\
$\quad$ Mean ( \pm s.D.) LOS per hospital stay (days) & $8 \cdot 33 \pm 15 \cdot 46$ & $7 \cdot 95 \pm 18 \cdot 30$ & $8 \cdot 03 \pm 17 \cdot 71$ \\
$\quad$ Median (range) (days) & $4(1-197)$ & $4(1-415)$ n.s. & $4(1-415)$ \\
(E) Total cost of admitted cases & $£ 1354865$ & $£ 5125442$ & $£ 6480828$ \\
Average cost per patient (E/A) & $£ 8162$ & $£ 15346$ & $£ 12962$ \\
Average cost per patient admitted (E/B) & $£ 13283$ & $£ 23088$ & $£ 20003$ \\
Average cost per admission (E/C) & $£ 4516$ & $£ 4840$ & $£ 4769$ \\
Median cost per patient admitted & $£ 6377$ & $£ 9326^{*}$ & $£ 8453$ \\
Median cost per patient & $£ 1498$ & $£ 3023^{*}$ & $£ 2394$ \\
\hline \hline
\end{tabular}

$\mathrm{HBV}$, Hepatitis B virus; $\mathrm{HBsAg}+, \mathrm{HBV}$ surface antigen positive; s.D., standard deviation.

Net costs were obtained from the Information Statistics Division website. Ninewells Hospital speciality group costs were used where possible, otherwise the average cost for Scotland was used. Where a patient moved to a different speciality during the same stay the daily costs for each speciality were halved on the day of the move.

Mann-Whitney test for immune patients $v s$. HBsAg + patients.

* $P<0 \cdot 05 ;$ n.s., not statistically significant.

medication costs. The cost of medication for a HBV patient varies from $£ 1095$ to $£ 8424$ per annum depending on disease severity and type of drug used [16].

The classification of patients was based only on HBV antibody and antigen status, but this is unlikely to have impacted on the prevalence and incidence estimates of diagnosis. The reason for this older classification is of course due to the long duration of this study during which time HBV DNA measurement was not routinely available. It does mean that some patients who were pre-core mutants, who should ideally have been quantified as a separate group, have been classified as 'carriers'.

\section{Diagnosis of liver condition}

The study produced the very interesting observation that HBV carriers had a lower rate of diagnosis of a liver condition than immune patients and as a consequence immune patients cost the health service more than those chronically infected with HBV, which is counter-intuitive. There are three possible explanations for this, one with positive connotations for the consequences of diagnosis and the other two more pragmatic. First, a patient knowing that they have chronic hepatitis may become more health aware and reduce risk behaviours compared to those who find they are immune and have no need to change behaviour. There is some evidence for such behavioural change in hepatitis $C$ patients [17]. The second, and perhaps more likely explanation, is that carriers might be less likely to be injecting drug users (IDUs) since IDUs acquire infection in adult life, usually present acutely, and eventually clear the virus. Hence, the lower rate of liver condition diagnosis in carriers may be related to the lower incidence of the many pathologies associated with IDUs, including overdose, suicide, endocarditis, hepatitis C and HIV. Third, healthy migrant selection may explain the lower rate of diagnosis in carriers, since those with a more aggressive form of HBV may be too unwell to achieve the economic status required to permit migration [18].

\section{Hospital costs}

The median cost per patient admitted for immune patients was twice that for chronic active and carrier patients. Again, this can be explained by the fact that the latter group of patients are likely to have had vertical infection and are predominantly immigrants, whereas immune patients are likely to have been 
infected in adulthood due to injecting drug use or risky sexual behaviour. Therefore the higher cost for immune patients is probably due to the conditions or diseases that are connected to the behaviours of these patients rather than HBV. For example, a total of 86 $(25.7 \%)$ of the 334 immune patients had HCV (22 before their initial HBV test and 64 post-test) compared to only seven $(5 \cdot 7 \%)$ of the 122 carriers (one before their initial HBV test and six post-test). However, it should be stressed that only $14.3 \%$ of the hospitalization costs for immune patients were related to liver disease admissions. It is unsurprising that the proportion of costs attributable to liver disease in the $\mathrm{HBsAg}+$ group is higher than in the immune group as the former have an active liver disease.

\section{CONCLUSIONS}

While the estimated incidence and prevalence of diagnosed HBV remained reasonably steady until 2003, the recent increase of immigrants may result or may have already resulted in an increase. The estimated incidence and prevalence of diagnosed immune HBV patients is steadily increasing. While this highlights an increase in screening and treatment for the virus, the costs associated with this group of patients are very high even though we did not include medication costs.

\section{ACKNOWLEDGEMENTS}

Our thanks go to the Health Informatics Centre (HIC), University of Dundee, a member of the MRC Health Services Research Collaboration, in particular Alison Bell, for anonymizing and abstracting the data. This study was funded by a grant from Gilead Sciences Ltd.

\section{DECLARATION OF INTEREST}

None.

\section{REFERENCES}

1. World Health Organization. Hepatitis B. Fact sheet No. 204 (http://www.who.int/mediacentre/factsheets/fs204/ en/). Accessed 6 December 2011.

2. Lavanchy D. Hepatitis B virus epidemiology, disease burden, treatment, and current and emerging prevention and control measures. Journal of Viral Hepatitis 2004; 11: 97-107.

3. Perz JF, et al. The contributions of hepatitis B virus and hepatitis $\mathrm{C}$ virus infections to cirrhosis and primary liver cancer worldwide. Journal of Hepatology 2006; 45 : 529-538.

4. Van Damme $\mathbf{P}$, Kane $\mathbf{M}$, Meheus A. Integration of hepatitis $\mathbf{B}$ vaccination into national immunisation programmes. Viral Hepatitis Prevention Board. British Medical Journal 1997; 314: 1033-1036.

5. Pendleton S, Wilson-Webb P. Rising curve: chronic hepatitis B Infection in the UK (http://www.hepb.org. uk/information/resources/rising_curve_chronic_hepati tis_b_infection_in_the_uk/rising_curve.pdf). Accessed 6 December 2011.

6. Metcalf M, et al. Health care costs associated with chronic hepatitisB. American Journal of Health-System Pharmacy 1999; 56: 232-236.

7. University of Dundee. Health Informatics Centre. About Tayside data (http://www.dundee.ac.uk/hic/ data/about/). Accessed 17 February 2012.

8. University of Dundee. The Health Informatics Centre (http://www.dundee.ac.uk/hic) Accessed 6 December 2011.

9. Steinke DT, Weston TL, Morris AD, et al. The epidemiology of liver disease in Tayside database: a population-based record-linkage study. Journal of Biomedical Informatics 2003; 35: 186-193.

10. Donnan PT, et al. Development of a decision support tool for primary care management of patients with abnormal liver function tests without clinically apparent liver disease: a record-linkage population cohort study and decision analysis (ALFIE). Health Technology Assessment 2009; 13(25).

11. Carstairs V, Morris R. Deprivation and mortality: an alternative to social class? Community Medicine 1989; 11: 210-219.

12. Evans JMM, MacDonald TM. Record-linkage for pharmacovigilance in Scotland. British Journal of Clinical Pharmacology 1999; 47: 105-110.

13. Information Services Division Scotland. Scottish Health Service costs 2009. File listings (http://www.isdscotlandarchive.scot.nhs.uk/isd/6480.html) Accessed 6 December 2011.

14. General Register Office for Scotland. In, out and net migration between NHS board areas and overseas by sex, 2001/02 to most recent (http://www.gro-scotland. gov.uk/files2/stats/migration/overseas-mig-flows-by-sexhb-2001-02-latest.pdf) Accessed 25 May 2012.

15. Steinke DT, et al. Epidemiology and economic burden of viral hepatitis: an observational population based study. Gut 2002; 50: 100-105.

16. Regional Drug and Therapeutics Centre, National Health Service. The use of adefovir dipivoxil for the treatment of chronic hepatitis B infection (http://www.nyrdtc.nhs. uk/docs/eva/adefovir.pdf) Accessed 6 December 2011.

17. Stoller EP, et al. Lay management of chronic disease: a qualitative study of living with Hepatitis C infection. American Journal of Health Behaviour 2009; 33: 376-390.

18. Gushulak BD, et al. Migration and health in Canada: health in the global village. Canadian Medical Association Journal. Published online: 28 June 2010. doi:10.1503/cmaj.090287. 